\title{
FUSOGENIC PROPERTIES OF UNCLEAVED SPIKE PROTEIN OF MURINE CORONAVIRUS JHMV
}

\author{
Fumihiro Taguchi, Toshio Ikeda, Keiichi Saeki, Hideyuki Kubo and Tateki \\ Kikuchi \\ National Institute of Neuroscience, NCNP, 4-1-1 Ogawahigashi, Kodaira \\ Tokyo 187, Japan
}

\begin{abstract}
We have tested the fusogenic properties of cleaved and uncleaved spike (S) protein of murine coronavirus (MCV) JHMV variant cl-2 by expressing the $\mathrm{S}$ protein by recombinant vaccinia viruses (RVVs). The amino acid sequence of the putative cleavage site of cl-2 S protein, Arg-Arg-Ala-Arg-Arg, was replaced by Arg-Thr-Ala-Leu-Glu by in vitro mutagenesis of cl-2 S gene. The RVVs having cl-2 S gene [RVV $t(+)$ ] or mutated cl-2 $\mathrm{S}$ gene [RVV t(-)] were tested for their ability to induce fusion as well as cleavability in DBT cells. After inoculation with RVV $t(+)$ onto DBT cells, the fusion formation was first observed at $8 \mathrm{~h}$ postinoculation (p.i.) and spread throughout the whole culture by $24 \mathrm{~h}$. In cells infected with RVV t(-), fusion appeared by $2 \mathrm{~h}$ and most of cells were fused by $30 \mathrm{~h}$ p.i. The $S$ protein and its cleavage products were detected in DBT cells expressing wild type $S$ protein. However, no cleavage products of the $S$ protein were detected in $R V V t(-)$ infected cells producing mutated $S$ protein, even though fusion was clearly visible. These results suggest that the cleavage event of JHMV-S protein of MCV is not a prerequisite for fusion formation, but that it enhances fusion.
\end{abstract}

\section{INTRODUCTION}

The S protein of MCV comprising the peplomer on the virion surface is 150 to $180 \mathrm{k}$ daltons, depending upon the virus 1,2 . This protein is multifunctional 2,3 . It is known that the $S$ protein is involved in the binding of virions to the receptor on susceptible cells 4 . Fusion of infected cells into polykaryocytes is also shown to be dependent upon S protein 2,5 . In addition. the major neutralizing epitopes exist on the $S$ protein. Furthermore, it is speculated that the $S$ protein is a major determinant of the virulence of the virus 6,7 .

It has been reported that a cleavage event is important for the fusogenic properties of $S$ protein 8,9 as in the cases of orthomyxoviruses and paramyxoviruses 10 . The cleavage of $S$ protein is supposed to be carried out by a host cell-derived trypsin like proteolytic enzyme. In the case of JHMV, the putative cleavage site is composed of the basic amino acid cluster of Arg-Arg-Ala-Arg-Arg 11, 12. On the other hand, it has been reported that the membrane anchoring subunit of cleavage product has no hydrophobic amino acid cluster that might function as a putative fusion peptide $2,11,12$. This differs from the situation of other viruses with fusion activity 10 . This finding suggests that mechanisms of coronavirus fusion formation may be different from that of the ortho- and paramyxoviruses. To delineate the mechanism of fusion formation by coronaviruses, it is of great importance to determine whether the cleavage of $\mathrm{S}$ protein is requisite for fusionability by the $\mathrm{S}$ protein. 


\section{RESULTS}

\section{Removal of the endoproteolytic cleavage site of $S$ protein}

The amino acid sequence 765 Arg-Arg-Ala-Arg-Arg 769, is predicted to be involved in the cleavage by host cell-derived proteolytic enzyme and this cleavage could occur between residues 769 and 770 (Fig. 1). We replaced this amino acid sequence by the Arg-Thr-AlaLeu-Glu sequence, predicted to be resistant to such an enzyme. Therefore, a mutant with this uncleavable peptide was created to test the effect of S protein cleavability on cell fusion. The site-directed mutation was carried out by making use of polymerase chain reaction (PCR). A pair of oligonucleotides were used to insert the mutation, one of which is a JHMTry(-), complementary sequence encoding a mutated amino acid sequence which can be obtained by three nucleotides substitution as shown in Fig. 1. The other oligonucleotide is genomic sense and corresponds to the sequence ca. 650 nucleotide upstream from JHMTry(-). After amplification by PCR, a 443 nucleotide fragment was obtained by cutting with $\mathrm{Cla}$ I and $\mathrm{Bal}$ 1. This was inserted in the wild type $\mathrm{S}$ gene where the corresponding wild type Bal I-Cla I 443 nucleotide fragment had been removed. The mutated $\mathrm{S}$ gene expected to encode the uncleavable $S$ protein was then inserted into the transfer vector $\mathrm{pSF}$ for obtaining the RVV. Finally, RVVs with the wild type $S$ gene $\{\mathrm{RVV} t(+)\}$ and mutated $S$ gene $\{\mathrm{RVV} t(-)\}$ were obtained.

\section{Fusion formation with wild type and mutated $S$ proteins expressed by RVVS}

DBT cells were infected with RVV $t(+)$ to produce wild type $S$ protein or $R V V t(-)$ to produce mutated $S$ protein at an m.o.i of 0.5 and observed for the presence or absence of fusion. Fused cells first appeared at about $8 \mathrm{~h}$ p.i. with RVV t(+). By $24 \mathrm{~h}$, more than 90 per cent of cells were involved in the fusion. In DBT cells infected with RVV $t(-)$ under the same condition, the fusion was also first observed at $12 \mathrm{~h}$ p.i. Thereafter, the fusion continued as that by $\mathrm{RVV} \mathrm{t}(+)$ (Fig. 2), except for a 2 to $4 \mathrm{~h}$ delay in appearance and development. The fusion produced by RVV $t(+)$ and $R V V t(-)$ was due to the expressed $S$ protein as fusion formation was prevented by monoclonal antibodies (MAbs) specific for cl$2 \mathrm{~S}$ protein (data not shown). These data clearly show that the wild type S protein as well as the mutated S protein containing Arg-Thr-Ala-Leu-Glu in place of Arg-Arg-Ala-Arg-Arg induced fusion in DBT cells.

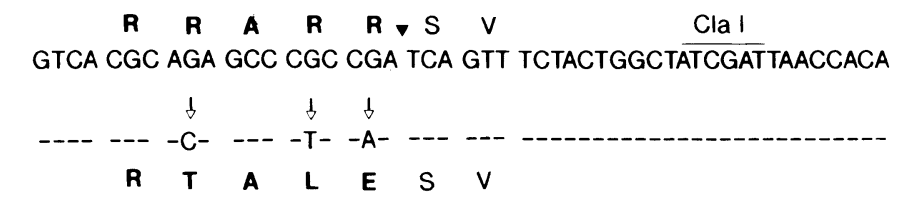

3' CAGT GCG TGT CGG GAG GTT AGT CAA AGATGACCGATAGCTAATTGGTGT 5' : JHM-TRY(-)

Figure 1. The amino acid and nucleotide sequences in the vicinity of the putative proteolytic cleavage site as well as the oligonucleotide (JHM-Try(-)) used to replace the cleavage site on wild type S protein. Black arrow shows the cleavage site.

\section{Analysis by immunoprecipitation and Western blotting of wild type and mutated $S$ proteins expressed by RVVs}

RVV $t(+)$ and $R V V t(-)$ as well as control vaccinia virus were used to infect DBT cells at an m.o.i. of 0.5 . At $14 \mathrm{~h}$ after the infection, when about $50 \%$ of RVV $t(+)$ infected cells and $20 \%$ of $\mathrm{RVV} t(-)$ infected cells were fused, the cells were pulse-labeled with ${ }^{35} \mathrm{~S}$ methionine for $30 \mathrm{~min}$ to detect the precursor $\mathrm{S}$ protein of an approximate molecular weight of $170 \mathrm{~K}$. Thereafter, the labeled proteins were chased for 1 to $3 \mathrm{~h}$ to detect $\mathrm{S}$ protein cleavage products. As shown in Fig. 3 , in the pulse-labeled samples, only one band 
corresponding to about $170 \mathrm{k}$ daltons was precipitated by the MAbs specific for cl-2 $\mathrm{S}$ protein in the lysates prepared from DBT cells infected either with RVV $t(+)$ or with RVV $\mathrm{t}(-)$. In the chase samples, S1 and S2 cleavage products were detected from RVV $\mathrm{t}(+)$ infected cells, but no such bands were found in the chased samples from RVV t(-) infected cells. Since the infected cells were labeled for $30 \mathrm{~min}$ only, it may have been that the incorporation of ${ }^{35} \mathrm{~S}$ methionine was insufficient for the detection of trace amounts of $S$ protein cleavage products by immunoprecipitation. Therefore, the presence of S1 and S2 was examined by more sensitive Western blotting with enhanced chemiluminescence. Here again only the $170 \mathrm{~K} \mathrm{~S}$ protein and no cleavage products were detected in $\mathrm{RVV} \mathrm{t}(-)$ infected DBT cells, while both the $170 \mathrm{~K}$ and its cleavage products were observed in cells infected RVV $t(+)$ infected cells (data not shown).
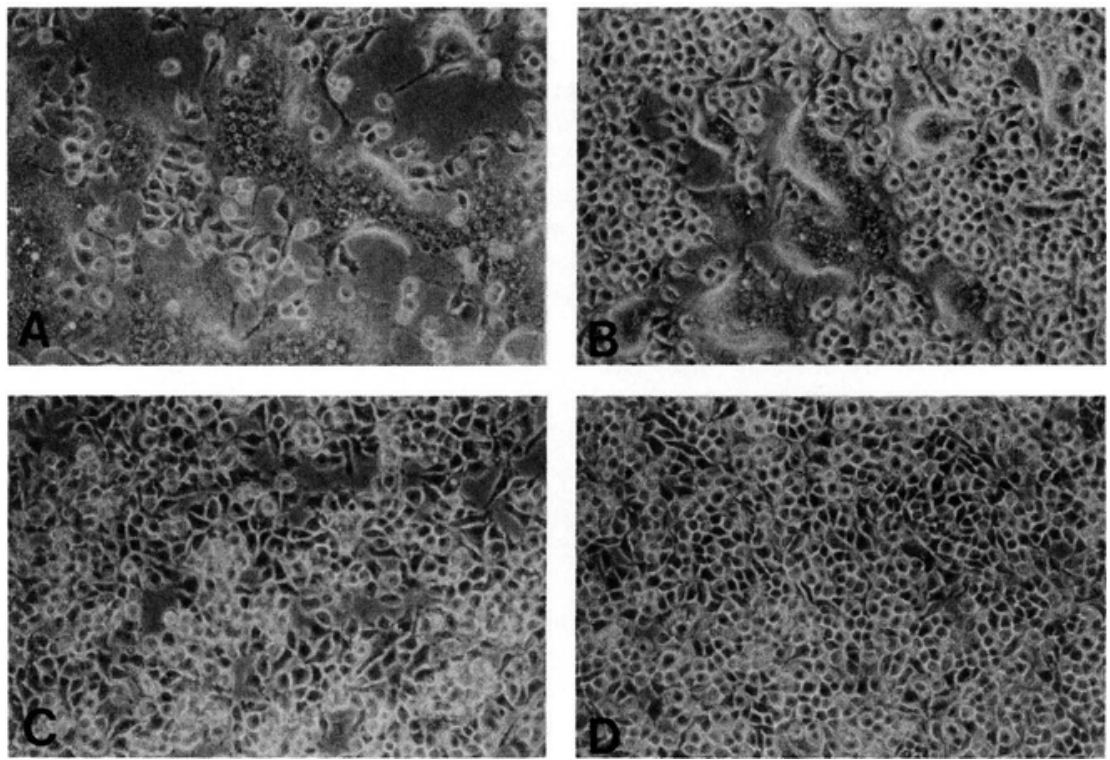

Figure 2. The fusion formation in DBT cells at $12 \mathrm{~h}$ after the infection with RVV $t(+)(A), \operatorname{RVV} t(-)$ (B) vaccinia virus (C) or mock infected DBT cells (D).

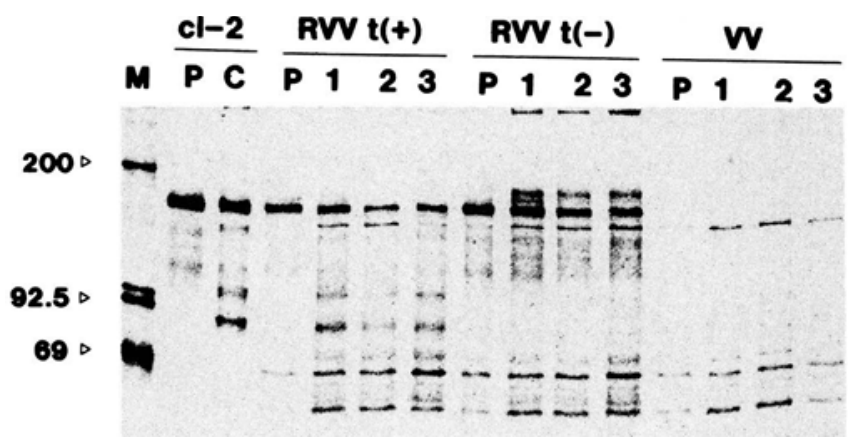

Figure 3. Immunoprecipitation of wild type and mutated $S$ proteins expressed in DBT cells by the infection with RVV t(+), RVV t(-) or vaccinia virus (VV). Infected cells were 30 min pulse-labeled at $14 \mathrm{~h}$ p.i. (P) and chased for 1 (1), 2 (2) and 3 (3) h. The $S$ protein produced in DBT cells infected with cl-2 (cl-2) was also precipitated. 


\section{DISCUSSION}

Fusion formation has been reported to be induced by orthomyxoviruses, paramyxoviruses and retroviruses 10 . The proteins involved in fusion formation by these viruses are envelope proteins which in most cases comprise the peplomer or spike of the virions ${ }^{10}$. The cleavage of the envelope protein is an absolute requirement for membrane fusion of cells infected with these viruses, which exposes the hydrophobic amino acid cluster at the $\mathrm{N}$ terminus of the membrane anchoring subunit of cleavage product 10 . This hydrophobic amino acid cluster designated as fusion peptide is believed to be a fusion active site.

The cleavage event was suggested to be an important step for fusion formation by coronaviruses from the facts that MCV with a fusion negative phenotype was rendered fusionable after treatment with proteolytic enzymes 9,13 . Also it has been reported that a correlation exists between the degree of cleavage or protein and the extent of fusion in different virus-cell systems ${ }^{8}$. All of these data are suggestive that the cleavage of the $S$ protein was a prerequisite for the fusion formation by $S$ protein of MCVs. This is analogous to similar mechanisms of fusion formation in other fusogenic RNA viruses. The results presented in this paper, however, indicate that fusion formation was caused by the uncleaved $S$ protein which contained a mutation in the putative cleavage site, implying that the cleavage of $S$ protein is not a prerequisite for the fusogenic activity of $S$ protein. The possibility, however, cannot completely be excluded that undetectable trace amounts of the cleaved $S$ protein were responsible for the fusion activity.

The data shown in the present paper are compatible with the idea that the mechanisms of fusion formation by coronavirus $S$ protein may differ from that of other viruses such as paramyxovirus, orthomyxovirus and retroviruses. This idea has been prompted by the facts that no hydrophobic amino acid cluster, homologuous to the fusion peptide of other fusogenic RNA viruses ${ }^{10}$, was found at the $\mathrm{N}$ terminus of the membrane anchoring chain $\mathrm{S} 2$, after cleavage of coronavirus $\mathrm{S}$ protein $2,12,14,15$. Semliki forest virus and Sindbis virus are capable of inducing fusion in infected cells after cleavage of the envelope protein and are known to have a stretch composed of 16 to 17 hydrophobic amino acids that constitute a putative fusion peptide in an internal position but not at the $\mathrm{N}$ terminus of the membrane anchoring chain 10 . However, in the S protein of JHMV cl-2 variant 12 as well as the $\mathrm{S}$ proteins of other coronaviruses such a large stretch of hydrophobic amino acid has not been detected 2,11, 11,15. It has been also documented by sequence analysis that some other coronaviruses, feline infectious peritonitis virus and porcine transmissible gastroenteritis virus, do not have the predicted proteolytic cleavage site on the S protein 2 . Nevertheless, fusion was found in cells infected with such viruses 16.

Little is known about the mechanisms of fusion formation by coronavirus $\mathrm{S}$ protein and there are only a few reports analyzing the relationship between the structure and fusion of the $\mathrm{S}$ protein in murine coronaviruses. To understand fusion mechanisms of coronaviruses, it is essential to locate the $\mathrm{S}$ protein fusion active site.

\section{Acknowledgments}

This research was partly supported by the grant provided by the Ichiro Kanehara Foundation.

\section{REFERENCES}

1. S. Siddell, H. Wege, and V. ter Meulen, J.gen.Virol. 64: 761-776 (1983)

2. W. Spaan, D. Cavanagh, and M.C. Horzinek, J.gen.Virol. 69: 2939-2952 (1988)

3. K.V. Holmes, E.W. Doller, and J.N. Behnke, Adv.Exp.Med.Biol. 142: 1331-142 (1981)

4. R.K. Williams, C. Jiang, and K.V. Holmes, Proc.Natl.Acad.Sci, USA, 88: 5533-5536 (1991) 
5. A.R. Collins, R.L. Knobler, H. Powell, and M. Buchmeier, Virology, 119: 358-371 (1982)

6. R.G. Dalziel, P.W. Lampert, J. Talbot, and M.J. Buchmeier, J.Virol. 59: 463-471 (1986)

7. Y. Matsubara, R. Watanabe, and F. Taguchi, Virus Res. 20: 45-58 (1991)

8. M.F. Frana, J.N. Behnke, L.S. Sturman, and K.V. Holmes, J.Virol. 56: $912-920$ (1985)

9. L.S. Sturman, C.S. Ricard, and K.V. Holmes, J.Virol. 56: 904-911 (1985)

10. J.M. White, Annu.Rev.Physiol. 52: 675-697 (1990)

11. I. Schmidt, M. Skinner, and S. Siddell, J.gen.Virol. 68: 47-56 (1987)

12. F. Taguchi, T. Ikeda, and H. Shida, J.gen.Virol. 73: 1065-1072 (1992)

13. H. Yoshikura, and S. Tejima, Virology 113: 503-511 (1981)

14. T.M. Gallagher, C. Escarmis, and M.J. Buchmeier, J.Virol . 65: 1916-1928 (1991)

15. S.E. Parker, T.M. Gallagher, and M.J. Buchmeier, Virology 173: 664-673 (1989)

16. R.J. De Groot, R.W. Vanleen,M.J.M. Dalderup, H. Vennema, M.C. Horzinek, and W.J.M. Spaan,Virology 171: 493-502 (1989) 